\title{
Intersubjectivité et métaphysique chez Merleau-Ponty
}

\author{
Keiichi Yahata
}

Université Osaka, JSPS

Le but de cet article est d'examiner une forme particulière d'intersubjectivité ainsi que son rapport avec la métaphysique, en analysant notamment un texte de Maurice Merleau-Ponty intitulé « Le roman et la métaphysique » (paru en 1945 et repris plus tard dans Sens et non-sens). Dans ce texte, alors qu'il commente un roman de Simone de Beauvoir en s'interrogeant sur une possible «liberté » entre des consciences comprises en un sens hégélien - liberté qui permettrait de découvrir le véritable sens de notre «existence »-, Merleau-Ponty dégage l'essence de la subjectivité en tant que «être-au-monde ». Il essaie également de cerner un nouveau sens pour le terme "métaphysique ", à savoir le «fonctionnement discordant de l'intersubjectivité humaine »".

C'est L'invitée, le premier grand roman de Beauvoir paru en 1943, que MerleauPonty analyse dans «Le roman et la métaphysique »" Dans ce roman, trois personnages (Françoise, Pierre et Xavière) entretiennent une étrange relation amoureuse qui aboutit à une fin belle et tragique. En s'intéressant à cette extraordinaire relation à trois de son propre point de vue, Merleau-Ponty entend trouver un «lien » essentiel entre le sujet et autrui, ainsi que situer dans ce lien même une « vraie morale », par-delà la morale vulgaire.

Précisons les trois étapes de cet article. Nous analysons d'abord un mode très particulier d'intersubjectivité propre aux deux personnages principaux de L'invitée : Françoise et Pierre. Nous dévoilons ensuite, à travers la « ruine » de cette intersubjectivité particulière, l'essence même du sujet comme "être-au-monde ». Nous tâchons de montrer enfin qu'un tel phénomène de "discordance » de l'intersubjectivité renvoie précisément chez notre philosophe à une redéfinition du sens même de la « métaphysique ».

\footnotetext{
${ }^{1}$ Maurice Merleau-Ponty, Sens et non-sens (désormais : SNS), Paris, Gallimard (coll. « Bibliothèque de philosophie »), [1948] 1996, p. 119.

${ }^{2}$ Merleau-Ponty cite ce même texte dans sa Phénoménologie de la perception pour aborder la « certitude » de notre cogito. Cf. Maurice Merleau-Ponty, Phénoménologie de la perception (désormais : PP), Paris, Gallimard (coll. « Tel »), [1945] 2002, p. 438.
} 


\section{1/ Intersubjectivité de la conscience}

Ce qui mérite tout d'abord d'être signalé dans L'invitée, c'est l'étrange relation amoureuse établie entre Françoise et Pierre : ce que Merleau-Ponty appelle l' « être à deux ». Et cet " être à deux », c'est Françoise qui en est le centre. Elle se dessine dans ce roman comme un personnage entièrement rationnel, croyant en la «toutepuissance du langage » : elle se sent située au cœur du monde et croit qu'elle n’a pas de corps. Françoise peut donc être qualifiée en termes philosophiques de personnage idéaliste ou solipsiste ${ }^{1}$. Merleau-Ponty écrit à son propos : « Tout ce qui arrive n’est que spectacle pour ce spectateur indestructible, impartial et généreux, tout n’est que pour elle $»^{2}$. Certes, du point de vue phénoménologique, la chose et autrui doivent essentiellement être ce qui me transcende, mais pour Françoise leur «transcendance » est littéralement et complétement "surmontée » ou « sublimée » par sa conscience même : « en elle coexistent tous les autres et le monde entier ».

Dès lors, il n'y a pour elle aucun « Autre » au sens propre (ou bien encore au sens lévinassien) du terme. Elle existe en quelque sorte comme une pure et simple « conscience » (idéaliste) et enveloppe en elle-même tous les autres et le monde. Il y a toutefois une exception à cet enveloppement idéaliste de Françoise : c’est son amant Pierre. Ceci étant, même si «sans doute Pierre n’est plus pour elle un objet dans son propre monde, un décor de sa vie, comme le sont les autres hommes » ${ }^{3}$, il « n’est pas davantage un Autre ». Françoise et Pierre forment ainsi ensemble un seul « être à deux », sans être chosifiés l'un par l'autre ni devenir « Autres » l'un pour l'autre.

Or, on pourrait dire que cet « être à deux » constitue dans un certain sens une sorte d'intersubjectivité. En effet, Françoise «a cru pouvoir [...] se vouloir en le [i.e. Pierre] voulant comme chacun veut autrui dans le règne des fins kantien »" Dans cet " être à deux », Françoise peut se rapporter à son partenaire comme elle se rapporte à elle-même. La distinction phénoménologique entre moi et autrui se voit ainsi assez affaiblie même si elle n’est pas entièrement supprimée.

Cet « être à deux » n'admet toutefois pas d'autres sujets que Françoise et Pierre. Les autres ne sont admis dans cette relation à la fois intersubjective et exclusive qu' «à titre d'invités » : la transcendance d'autrui (mais aussi de la chose) en est totalement exclue ${ }^{5}$. Dans la mesure où il n'accepte aucun autre sujet, cet « être à

\footnotetext{
${ }^{1}$ « Je demeure le centre du monde. Je suis cet être agile qui circule à travers le monde et l'anime de part en part. Je ne peux pas sérieusement me confondre avec cette apparence que j’offre aux autres. Je n’ai pas de corps » (Maurice Merleau-Ponty, SNS, p. 38).

${ }^{2}$ Ibid., p. 38.

${ }^{3}$ Ibid., p. 39.

${ }^{4}$ Ibid., p. 42.

5 «Si Françoise et Pierre suscitent autour d'eux tant d'envies et même de haines, n'est-ce pas que les autres se sentent exclus par ce prodige à deux têtes, jamais accueillis par eux, mais toujours trahis par Françoise avec Pierre, par Pierre avec Françoise ? » (Ibid., p. 40).
} 
deux » apparemment intersubjectif est loin de correspondre à ce que Merleau-Ponty appelle l' «intersubjectivité corporelle » (ou plus simplement l' «intercorporéité »), intersujectivité capable d'intégrer tous les autres dans un seul système de Corps (ou de Chair) en tant que ses membres ou ses organes. C'est pourquoi cet " être à deux » renvoie plutôt à une intersubjectivité de la conscience (basée seulement sur la « raison » et le « langage »). Par rapport à l'intercorporéité, il s’agit dès lors d’une pseudo-intersubjectivité. En conséquence, l' « être à deux » caractérisant la relation de Françoise et Pierre est marqué - paradoxalement voire contradictoirement par une intersubjectivité solipsiste qui n'admet pas d'autres sujets que ses deux constituants.

\section{2/ « Ruine » de l’intersubjectivité de la conscience et l'être-au-monde}

Dans cette pseudo-intersubjectivité constituée par Françoise et Pierre intervient également un troisième personnage, $\mathrm{Xavière}^{1}$, qui va ruiner leur « être à deux ». C’est précisément cette " ruine » de l'intersubjectivité de la conscience qui permet de dévoiler le rapport fondamental entre le sujet et le monde. Le déroulement de cette ruine est d'ailleurs rythmé par deux étapes. Dans un premier temps, lorsque Xavière enlève à Françoise son amant, cette dernière se trouve détachée de son monde : « elle n'est plus comme par un privilège naturel au cœur des choses : il y a un centre du monde d'où elle est exclue, c'est l'endroit où Pierre et Xavière doivent se retrouver $»^{2}$. On a affaire ici à une sorte de détachement du sujet par rapport au monde qui donne lieu à la fragmentation du temps historique. Dans la ruine de son « être à deux », Françoise est elle-même exclue du cœur du monde où elle croyait demeurer.

\footnotetext{
${ }^{1}$ « Xavière remet en question toutes les conventions par lesquelles Françoise et Pierre avaient cru rendre leur amour invulnérable » (Ibid., p. 41). Xavière est décrite dans ce roman comme un personnage qui contraste très nettement avec Françoise. Alors que cette dernière représente "une confiance absolue dans le langage et dans les décisions rationnelles, une existence qui se vide à force de se transcender ", Xavière est associée à un "immédiat fermé sur lui-même, en deçà de toute parole et de tout engagement» (Ibid., p. 50-51), ou est tout simplement qualifiée d'« égoïste » : elle "ne se prête ou ne se donne jamais à aucun projet, [...] elle ne sacrifie jamais l'immédiat, elle ne sort jamais de l'instant, elle adhère toujours à ce qu'elle éprouve », de telle sorte qu' « on vit à côté d'elle, [mais] on ne vit pas avec elle » (Ibid., p. 45-46). Xavière ne constitue pas même un "être à deux " avec Pierre comme le faisait Françoise, et on pourrait dire de ce fait qu'elle est très éloignée de l'intersubjectivité. De plus, à la différence de Françoise, Xavière n'existe pas comme une conscience pure, mais elle s'attache («adhère ») toujours à la dimension de son expérience immédiate. L'un des buts de Merleau-Ponty dans « Le roman et la métaphysique » consiste à rechercher « entre ces deux limites où elle [l'existence] périt », cette " existence totale qui est la décision par laquelle nous entrons dans le temps pour y créer notre vie » (Ibid., p. 51).

${ }^{2}$ Ibid., p. 42.
} 
Or, comme Merleau-Ponty l'a déjà souligné dans Phénoménologie de la perception, le sujet et le monde s'opposent en réalité l'un à l'autre dans leur essence même. En effet, tandis que le sujet est caractérisé par un "non-être " (plus exactement un « réduit de non-être »), le monde nous apparaît — dans l'un de ses aspects du moins - comme un monde ontique plein de choses (ce que le philosophe appelle le «monde naturel »). Ainsi, entre le sujet comme "être-au-monde » et ce monde auquel il est inhérent surgit toujours un écart fondamental ${ }^{1}$. Au dire de MerleauPonty, à l'instar de Malebranche, le monde est un « ouvrage inachevé » ${ }^{2}$, mais ce qui le rend ainsi c'est le sujet même en tant que « réduit de non-être » dans ce monde de l'être. Autrement dit, l'homme «est à la lettre un défaut dans le diamant du monde $»^{3}$. Lorsque la pseudo-intersubjectivité de Françoise et de Pierre tombe en ruine et qu'elle est exclue de son propre monde, l'écart entre le sujet et le monde se manifeste en tant que tel. Le monde — qui était jusque-là « humain » — se sépare dès lors du sujet lui-même et (re)devient de ce fait le monde «naturel » (ou «sauvage ») des choses — c'est d'ailleurs justement ce monde « naturel » ou « inhumain », détaché de tout humain et " qui interdit toute effusion humaine »" que Cézanne s'efforce de peindre et dans lequel il voudrait s'enfuir d'après MerleauPonty. C'est avec autrui que ce monde recommence à transcender radicalement le sujet $^{5}$. Françoise, qui voulait autrui (Pierre) comme elle se voulait elle-même, est donc non seulement rejetée par le monde et par autrui mais également exclue d'ellemême ; elle découvre ainsi, au-delà du « règne des fins kantien », « le soi hégélien qui poursuit la mort de l'autre ${ }^{6}$.

Dans un deuxième temps, la ruine de la pseudo-intersubjectivité advient lorsque Françoise est atteinte de maladie et entre à l'hôpital. Merleau-Ponty soutient alors que pour Françoise « le centre du monde est pour le moment dans cette chambre [:] la grande affaire de la journée, c’est la température, l'examen radioscopique, le premier repas qu'on va lui donner. Tous les objets ont repris mystérieusement leur

\footnotetext{
${ }^{1}$ Dans sa Phénoménologie de la perception, Merleau-Ponty caractérise notre subjectivité comme un « réduit de non-être » et souligne qu'elle ne croise jamais le monde de l'être, lequel est plein de choses (Maurice Merleau-Ponty, PP, p. 458 et p. 518). À notre avis, c'est dans le phénomène de la " parole parlante » que ce «non-être » de la subjectivité se transpose en «être » du monde. À ce propos, voir les premier et deuxième chapitres de notre thèse de doctorat : La problématique de l'expression dans la philosophie de Maurice Merleau-Ponty (version open acces : http://tel.archives-ouvertes.fr/tel-00830298/).

${ }^{2}$ Maurice Merleau-Ponty, SNS, p. 49.

${ }^{3}$ Ibid., p. 57.

${ }^{4}$ Ibid., p. 22.

${ }^{5}$ « Même les choses qui m'entourent me dépassent à condition que j'interrompe mon commerce habituel avec elles et que je les retrouve, en deçà du monde humain ou même vivant, sous leur aspect de choses naturelles " (Ibid., p. 37). Les choses apparaissent alors à Françoise comme des " débris étranges dont elle n’a plus la clef » (Ibid., p. 42).

${ }^{6}$ Ibid., p. 42.
} 
valeur ${ }^{1}$. Autant dans la première étape surgit un détachement entre le sujet et le monde et recommence de ce fait la transcendance radicale de la chose et d'autrui, autant dans cette seconde étape toutes les choses — devenues désormais des « débris étranges » du monde — « reprennent leur valeur » pour le sujet. Cela ne signifie pas pour autant que leur transcendance est nivelée ou supprimée dans la pure conscience réflexive, mais au contraire que le sujet est lui-même chosifié : Françoise «s'est repliée de son monde humain où elle souffrait dans le monde naturel où elle trouve une paix glacée ». Le sujet, qui est proprement caractérisé par le "non-être » et qui se situe à un niveau tout autre que celui du monde de l'être, se chosifie (s'ontifie) lui-même, s'assimile au monde de l'être et devient un objet exposé au regard des autres sujets. L'écart fondamental entre le sujet et le monde se voit ainsi comblé ; aussi bien la transcendance de la chose que celle d’autrui ${ }^{2}$ sont supprimées, quoique de manière différente.

La « ruine » de l' " être à deux » de Françoise et de Pierre, provoquée directement par Xavière et réalisée progressivement dans ces deux étapes — entre lesquelles intervient la «mort» comme une sorte de valeur limite idéale de l'existence suspendant tous les projets existentiels ${ }^{3}$ - , nous permet de mieux saisir l'essence même de l' « être-au-monde ». Premièrement, l' « être à deux » n'est qu'une pseudointersubjectivité constituée de façon dogmatique par la conscience (idéaliste et solipsiste) ; deuxièmement, et de manière plus décisive, il existe un écart fondamental entre le sujet non-ontique et le monde ontique (ou chosique $)^{4}$ : dans la mesure où cet écart se manifeste en tant que tel, le sujet se rend compte qu'il y a une "solitude " ${ }^{5}$ absolue ou une « complicité avec soi-même » dans son existence. Lorsque la pseudo-intersubjectivité de la conscience tombe en ruine, le sujet est d'emblée exclu de lui-même ainsi que rejeté par autrui. Ceci étant, il trouve au sein de cette exclusion un «lien » plus intime et plus profond avec lui-même qui pourrait correspondre à la "généralité de ma subjectivité » qui compense la « généralité du corps » selon la terminologie de Phénoménologie de la perception ${ }^{6}$. Puisque ce « lien » jamais rompu renvoie à la «solitude » indépassable de notre existence même, il constitue, selon nous, le point d'arrivée auquel aboutit l'intersubjectivité de la conscience de Françoise.

\footnotetext{
${ }^{1}$ Ibid., p. 44.

${ }^{2}$ Les autres se déshumanisent alors et «sont intermittents comme des personnages de théâtre » (Ibid., p. 44).

${ }^{3}$ Ibid., p. 43.

${ }^{4}$ D'après Merleau-Ponty, c'est parce qu'elles décrivent cet écart fondamental entre le sujet et le monde que l'on reproche souvent aux œuvres de Sartre d'être pleines de «laideurs ». Cela signifie en fait qu' « il met la valeur de l'homme dans son imperfection» (Ibid., p. 57). Mais, comme nous le verrons plus tard, cette « laideur » constitue plutôt la condition de la « vraie morale ».

${ }^{5}$ Ibid., p. 44.

${ }^{6}$ Maurice Merleau-Ponty, PP, p. 411.
} 
Or, avant de traiter dans la section suivante du rapport entre ce phénomène de la « ruine » de l’intersubjectivité et la «métaphysique » au sens merleau-pontien, abordons brièvement le rôle du " corps » dans cette ruine. Le lien intersubjectif noué entre Françoise et Pierre n'est pas corporel. Ici, le «corps » contribue plutôt à sa ruine : « pour la première fois elle [Françoise] a le sentiment d'être son corps, alors qu'elle se croyait une conscience ${ }^{1}$. Ayant constitué un «être à deux » heureux avec Pierre par sa pure conscience, Françoise découvre " pour la première fois » son propre corps dans l'effondrement de cet « être à deux ». Ce « corps » ainsi découvert ne donne toutefois pas lieu à un nouveau lien intersubjectif (corporel) entre Françoise et son amant ; il la met au contraire en dehors de sa pure conscience et dans la position donc d'un objet du monde (naturel ou chosique). Par conséquent, loin de désigner exclusivement l'expérience syncrétique propre à la dimension de la «Chair» ou de l' «intercorporéité », le «corps» pousse le sujet comme pure conscience vers son extériorité, l'exposant ainsi au regard des autres. C'est pourquoi ce dont il est ici question n’est pas le «corps phénoménal » mais le «corps objectif ». Au lieu de constituer le « véhicule » de l'être-au-monde ${ }^{2}$, le «corps » est le signe de la «discordance » entre le sujet et le monde : ce n’est qu'après avoir été exclu du monde que le sujet découvre son propre corps. Aussi la «ruine » de l’intersubjectivité met-elle en relief le double aspect de notre corps.

\section{3/ Intersubjectivité et métaphysique}

Nous avons vu que l'analyse merleau-pontienne de L'invitée remet en cause aussi bien le statut du sujet comme «être-au-monde » que son rapport au monde, tout en insistant particulièrement sur la « ruine » ou la " discordance » de l'intersubjectivité humaine. Selon Merleau-Ponty, dans la mesure où l'ouvrage de Beauvoir cherche à redéfinir le rapport du sujet au monde comme un au-delà de l'être-au-monde, ce dernier s’inscrit nécessairement dans la "littérature métaphysique ». Nous tâchons de clarifier dans cette dernière section le sens du terme « métaphysique » tel qu'il est employé par Merleau-Ponty, de même que la relation établie par ce dernier entre l'intersubjectivité et la métaphysique.

Qu'est-ce que la métaphysique pour Merleau-Ponty ? Un passage du texte intitulé « Le métaphysique dans l’homme » dans Sens et non-sens permet de répondre sans équivoque à cette question:

Or la métaphysique, réduite par le kantisme au système des principes que la raison emploie dans la constitution de la science ou de l'univers moral, radicalement contestée, dans cette fonction directrice, par le positivisme, n’a pas cessé,

\footnotetext{
${ }^{1}$ Ibid., p. 43.

${ }^{2}$ Maurice Merleau-Ponty, PP, p. 163.
} 
cependant, de mener dans la littérature et dans la poésie comme une vie illégale, et les critiques l'y retrouvent aujourd'hui ${ }^{1}$.

Même si, à l'opposé des conceptions classiques, Merleau-Ponty insiste sur le fait qu'une "métaphysique en acte » apparaît tout d'abord dans les sciences humaines contemporaines — notamment dans la «psychologie de la forme », la « linguistique », la «sociologie » et «l'histoire » qu'il aborde d'une manière tout à fait innovante - , nous nous interrogeons davantage sur sa nouvelle conception de la « métaphysique » elle-même.

Selon lui, alors que la métaphysique classique et traditionnelle « a pu passer pour une spécialité où la littérature n’avait que faire », qu’ « elle a fonctionné sur un fond de rationalisme incontesté » et qu' « elle était persuadée de pouvoir faire comprendre le monde et la vie humaine par un agencement de concepts $»^{2}$, cette nouvelle « métaphysique » est affranchie de la « construction de concepts par lesquels nous essaierions de rendre moins sensibles nos paradoxes » : elle se caractérise essentiellement par «l'expérience que nous en [de nos paradoxes] faisons dans toutes les situations de l'histoire personnelle et collective ${ }^{3}$ ainsi que par «l'exploration délibérée de ce monde avant l'objet de science auquel la science se réfère ${ }^{4}$. Autrement dit, tout en restant en deçà de « quelque au-delà » de l'être empirique de l'homme — «Dieu » ou la « Conscience » —, la « métaphysique » de notre temps vise à décrire le rapport fondamental du sujet au monde et à autrui («avant l'objet de science », même si il y a de la métaphysique dans la science). Étant donné que pour Merleau-Ponty la « métaphysique » s’inscrit dans la « philosophie phénoménologique ou existentialiste » qui «se donne pour tâche [...] de formuler une expérience du monde, un contact avec le monde qui précède toute pensée sur le monde ${ }^{5}$, elle opère au sein des expériences pleines de "paradoxes » et de «discordances » caractérisant toutes les situations de l'histoire personnelle et collective.

Dans cette conception inédite de la «métaphysique » (humanisée, pour ainsi dire), l' « intersubjectivité » joue un rôle important. En effet, selon Merleau-Ponty l'un des objectifs majeurs de cette nouvelle «métaphysique » touche précisément à la possibilité de «vérifier toujours à nouveau le fonctionnement discordant de l'intersubjectivité humaine ${ }^{6}$. La raison pour laquelle L'invitée de Beauvoir est considéré par lui comme faisant partie de la «littérature métaphysique " s’avère donc évidente. Aux antipodes du processus de constitution de l'intersubjectivité à

\footnotetext{
${ }^{1}$ Maurice Merleau-Ponty, SNS, p. 102.

${ }^{2}$ Ibid., p. 35.

${ }^{3}$ Ibid., p. 117.

${ }^{4}$ Ibid., p. 118.

${ }^{5}$ Ibid., p. 36.

${ }^{6}$ Ibid., p. 119.
} 
partir de l'expérience de soi (à l'instar notamment de Husserl), Merleau-Ponty aborde le rapport fondamental entre l'humain et le monde à travers la « ruine » ou la « discordance » de cette relation même, telles qu'elles sont décrites dans l’ouvrage de fiction en question. Nous en venons ainsi au sens de sa « métaphysique ».

Il faut toutefois préciser que cette «métaphysique » ne met pas uniquement en avant l'aspect négatif du phénomène de l'intersubjectivité. Nous avons beau constater que, dans la mesure où « il n'y a plus de nature humaine sur laquelle on puisse se reposer ${ }^{1}$, la «littérature métaphysique » de Sartre et de Beauvoir constitue à première vue " une littérature amorale », il n'en demeure pas moins que cette littérature vise à décrire le processus même de l'apparition de l'humain (le « sujet » ou le «sens ») sur fond de monde inhumain, c'est-à-dire sur la "racine préhumaine » ${ }^{2}$ que Merleau-Ponty aborde par le biais notamment du «fonctionnement discordant de l'intersubjectivité humaine ». Si l'œuvre de Sartre et de Beauvoir soulève bien souvent, de la part des critiques littéraires, des «reproches d'immoralité », c'est parce qu'ils n'en retiennent que la « laideur » propre à l'écart ou au décalage entre l'humain (le sujet) et l'inhumain (le monde), ce qui les empêche de reconnaître que la « littérature métaphysique » de Sartre et de Beauvoir tente de mettre au jour la naissance de l'humain sur fond d'inhumain. Leur œuvre esquisse en ce sens une « vraie morale » au-delà de la morale vulgaire.

À l'instar de Sartre, Beauvoir a essayé de décrire l' « imperfection » de l'homme — l' « imperfection fondamentale par laquelle il est et est seul capable de se faire » ${ }^{3}$. Ceci étant, il ne s’agit pas seulement dans leur «littérature métaphysique » d'accorder une priorité à cette « imperfection » de l'homme dans le but de relativiser sa prétendue perfection et de rejeter tout humanisme comme étant la « religion de l'homme achevé » ${ }^{4}$. Au lieu de prendre les divers objets humains comme «tout faits » — « comme des conséquences sans prémisses et comme s’ils allaient de soi » - , la « conscience métaphysique » dont Merleau-Ponty parle — conscience qui n'est pas au-delà de notre monde mais toujours en deçà — « redécouvre leur étrangeté fondamentale pour moi et le miracle de leur apparition $»^{5}$. De plus, cette conscience « est elle-même, par-delà le monde plat de la conscience habituée et

\footnotetext{
${ }^{1}$ Ibid., p. 37.

${ }^{2}$ Ibid., p. 60.

${ }^{3}$ Ibid., p. 58.

${ }^{4}$ Ibid., p. 57.

${ }^{5}$ Ibid., p. 115. Parce qu'elles restituent « leur transcendance et leur étrangeté originaires » (Ibid., p. 119) à certains phénomènes, cette conscience et cette littérature nous détachent de l'«attitude naturelle » - dans laquelle nous nous croyons « en présence d'un monde et d'un temps que notre pensée survole et dont elle peut à volonté considérer chaque partie sans en modifier la nature objective » (Ibid., p. 113) — et sont alors considérées comme "métaphysiques », c'est-à-dire comme «transnaturelles». Le terme "méta-physique » renvoie donc dans ce contexte à un au-delà de l'attitude naturelle.
} 
endormie, la connexion vivante de moi avec moi et de moi avec autrui » ${ }^{1}$. La «métaphysique » vise somme toute à saisir, en deçà de tout ce qui constitue l'humain, le moment où cet humain nous apparaît pour la première fois ; elle vise aussi à retrouver au sein même de ce moment le lien le plus profond avec le monde, avec autrui et avec soi-même. En décrivant volontairement dans leur œuvre littéraire ce qui n'est pas humain, Sartre et Beauvoir ont tous deux cherché à mettre en place une telle «métaphysique ».

\section{Conclusion}

Ce travail nous a permis de mettre en avant la nouvelle manière dont le sujet se rapporte au monde, à autrui et à soi-même ainsi que la nouvelle notion de «métaphysique » mise en œuvre par Merleau-Ponty dans certains textes des années 40 parus dans Sens et non-sens. Nous tenons pour conclure à faire une remarque concernant son existentialisme.

C'est parce que Merleau-Ponty est un philosophe existentialiste au même titre que Sartre que la puissance immanente au «sujet » ou au "sens » occupe une place fondamentale dans sa pensée. Cela ne veut pas dire pour autant que nous avons affaire à un humaniste naïf. Comme nous l'avons souligné à plusieurs reprises, c'est le moment où surgissent pour nous le sujet et le sens — leur « état naissant » — qui est au cœur du projet philosophique merleau-pontien, moment que Merleau-Ponty nommera plus tard l' « institution » : l' « événement » au cours duquel le sujet met en place un acte singulier pour créer non seulement un nouveau sens mais la condition — l'historicité - même de cet acte. Afin d'y parvenir, au lieu de se diriger directement vers l'humain, Merleau-Ponty se situe d'abord auprès de l'inhumain. La véritable signification de son existentialisme y est suspendue : l' " existence » consiste en un mouvement allant de l'inhumain (le non-sens) vers l'humain (le sens), ou bien encore en une tension entre les deux. Le sens de notre existence se dessine toujours par rapport au non-sens et sur fond d'inhumain.

D'après Merleau-Ponty, l'existentialisme ne rejoint jamais le simple humanisme qui n'est quant à lui qu'une «religion de l’homme achevé ». C’est pourquoi son existentialisme est fondé sur une «racine » inhumaine ou pré-humaine, là où l'homme reste toujours " inachevé » tout comme le monde lui-même. Cet existentialisme inhumain n'est toutefois pas anti-humain : «seul un homme justement est capable de cette vision qui va jusqu'aux racines, en deçà de l'humanité constituée $»^{2}$. Merleau-Ponty ne se passe pas de l'humain. C'est plutôt toujours à travers l'humain, et pour le rechercher, qu'il aborde l'inhumain comme fondement

\footnotetext{
${ }^{1}$ Ibid., p. 117.

${ }^{2}$ Ibid., p. 22.
} 
de l'humain. L'inhumain — c'est-à-dire l'existentialisme — nous permet de mieux saisir ce qu'il y a de plus humain dans notre monde. 